\title{
Vestibulopatia por lesão endovascular em cateterismo de urgência
}

\section{Vestibular system paresis due to emergency endovascular catheterization}

\author{
Lucinda Simoceli', Danilo Anunciatto Sguillar ${ }^{2}$, Henrique Mendes Paiva Santos², Camilla Caputti². \\ 1) Doutora em Otorrinolaringologia pela FMUSP. Responsável pelo Setor de Otologia do Hospital Beneficência Portuguesa de São Paulo. \\ 2) Residente do Segundo Ano de Otorrinolaringologia do Hospital da Beneficência Portuguesa de São Paulo. \\ Instituição: Hospital Beneficência Portuguesa de São Paulo. \\ São Paulo / SP - Brasil. \\ Endereço para correspondência: Danilo Anunciatto Sguillar - Avenida Paes de Barros, 1425, apto 122 - São Paulo / SP - Brasil - CEP: $03115-001$ - Telefone: (+55 11) \\ 3288-0899-E-mail: danilo_sguillar@hotmail.com \\ Artigo recebido em 14 de Março de 2010. Artigo aprovado em 16 de Maio de 2010.
}

\section{RESUMO}

Objetivo: O objetivo deste relato de caso é descrever uma causa incomum de vestibulopatia periférica associada à perda auditiva unilateral em paciente idoso pós- cateterismo de urgência.

Relato de caso: Paciente do gênero masculino, 82 anos, submetido à correção de aneurisma roto de aorta abdominal, no intra-operatório sofreu infarto agudo do miocárdio necessitando de angioplastia primária. Após alta hospitalar refere queixa de hipoacusia acentuada à direita e vertigem incapacitante, sem sinais neurológicos focais. Ao exame clínico otorrinolaringológico apresentava: Teste de Weber lateralizado para a esquerda, nistagmo espontâneo para a esquerda , marcha oscilante, leve disbasia e ataxia, índexnariz e diadococinesia normais, Teste de Romberg com oscilação sem queda e Fukuda com desvio lateral para a direita. O exame audiométrico evidenciava anacusia à direita e perda neurossensorial à esquerda em agudos, arreflexia vestibular à direita na prova calórica e, na tomografia computadorizada dos ossos temporais e tronco-encefálico, presença de haste metálica atravessando o osso temporal direito, a partir da veia jugular interna e bulbo jugular, atravessando os canais semicirculares posterior, superior e vestíbulo, projetando-se em lobo temporal. O diagnóstico radiológico foi lesão traumática por guia endovascular metálico durante cateterismo de urgência e a conduta, considerando que o paciente não havia compensado o equilíbrio, foi reabilitação vestibular.

Conclusão: Queixas de tontura no paciente idoso devem ser criteriosamente avaliadas diante do seu histórico clínico patológico pois os antecedentes de doenças e tratamentos prévios, em geral, direcionam as hipóteses diagnósticas porém podem trazer alterações inesperadas.

Palavras-chave: vestíbulo do labirinto, cateterismo cardíaco, emergências.

\section{SUMMARY}

Objective: The objective of this story of case is to describe an uncommon cause of associated peripheral Vestibulopathy to the unilateral auditory loss in aged patient after catheterization of urgency.

Story of case: Patient of the masculine sort, 82 years, submitted to the correction of abdominal ragged aneurism of aorta, in the intra-operative suffered heart attack acute from the myocardium needing primary angioplasty. High after hospital it relates to complaint of accented hearing loss to the right and crippling vertigo, without focal neurological signals. To the otorhinolaryngological clinical examination it presented: Test of Weber lateralized for the left, spontaneous nystagmus for the left, marches rocking, has taken normal disbasia and ataxia, index-nose and diadochokinesia, Test of Romberg with oscillation without fall and Fukuda with lateral shunting line for the right. The audiometric examination evidenced deafness to the right and sensorineural loss to the left in sharps, areflexia initial to the right in caloric test e, the computerized tomography of the secular bones and brainstem, presence of metallic connecting rod crossing the right secular bone, from the vein internal jugular vein and bulb jugular vein, crossing the posterior, superior and vestibule semicircular canals, projecting itself in temporal lobe. The radiological diagnoses was traumatic injury for guide to endovascular metallic during catheterization of urgency and the behavior, considering that the patient had not compensated the balance, it was vestibular rehabilitation. Conclusion: Complaints of giddiness in the aged patient must be closely evaluated of its pathological clinical description because the antecedents of illnesses and previous treatments, in general, direct the diagnostic hypotheses however they can bring unexpected alterations.

Keyword: vestibule of the labyrinth, cardiac catheterization, emergencies. 


\section{INTRODUÇÃO}

O equilíbrio corporal depende da integração dos sistemas vestibular, visual e proprioceptivo $(1,2)$. Dois fatores relacionam-se com as alterações de equilíbrio no idoso: o aumento progressivo do envelhecimento populacional e as doenças próprias da idade (2). Dentre elas, destacam-se a insuficiência vertebro-basilar, podendo ser representada por lesões isquêmicas, embólicas, dissecantes ou traumáticas (3), alterações do sistema nervoso central, a síndrome de desequilíbrio do idoso, alterações metabólicas/hormonais e a vertigem postural paroxística benigna (VPPB) (4). Clinicamente, as alterações do equilíbrio caracterizam-se por tontura, vertigem, desequilíbrio e queda e estima-se que a prevalência na população idosa chegue a $85 \%(5,6)$.

O objetivo deste relato de caso é descrever uma causa incomum de vestibulopatia periférica associada à perda auditiva unilateral em paciente idoso pós- cateterismo de urgência.

\section{Relato de CAso}

Paciente do gênero masculino, 82 anos, com antecedentes de hipertensão arterial sistêmica e dislipidemia em tratamento medicamentoso, foi submetido à cirurgia cardiovascular em setembro/2000 devido um aneurisma roto de aorta abdominal. No intra-operatório sofreu infarto agudo do miocárdio necessitando de angioplastia primária. Desde então, apresentou história de vertigem aos movimentos rápidos da cabeça e desequilíbrio às mudanças de decúbito como sentar e levantar e à deambulação. Permaneceu quinze dias em UTI e recebeu alta hospitalar após um mês de internação com as queixas descritas e sem sinais neurológicos focais. Fez uso de dicloridrato de flunarizina por 5 anos orientado pelo cardiologista e manteve a queixa de desequilíbrio e hipoacusia. Ao exame clínico otorrinolaringológico apresentava: Teste de Weber lateralizado para a esquerda, nistagmo espontâneo para a esquerda, marcha oscilante, leve disbasia e ataxia, índexnariz e diadococinesia normais, Teste de Romberg com oscilação sem queda e Fukuda com desvio lateral para a direita.

O exame audiométrico evidenciava anacusia à direita e perda neurossensorial à esquerda nas frequências 4, 6 e $8 \mathrm{kHz}$, e o exame otoneurológico evidenciava presença de nistagmo espontâneo horizontal para a direita com VACL de 3 graus/segundo, Testes oculomotores sem alterações e arreflexia vestibularà direita na prova calórica. Foi realizada tomografia computadorizada dos ossos temporais e tronco-encefálico, que mostrou presença de haste metálica atravessando o osso temporal direito, a partir da veia jugular interna e bulbo jugular, atravessando os canais semicirculares posterior, superior e vestíbulo, projetandose em lobo temporal (Figuras 1 e 2). O diagnóstico radiológico foi lesão traumática por guia endovascular metálico durante cateterismo de urgência e a conduta, considerando que o paciente não havia compensado o equilíbrio, foi reabilitação vestibular. Após 40 dias de terapia o paciente referia melhora de $90 \%$ e após 3 meses de terapia, apresentou remissão completa dos sintomas de desequilíbrio. Atualmente encontra-se em acompanhamento radiológico há 3 anos sem mobilização da haste metálica.

\section{DISCUSSÃO}

A maioria dos autores concorda que, em se tratando de alterações do equilíbrio na população idosa, deve-se lembrar da etiologia multifatorial, uma vez que existem inúmeros fatores de risco concorrentes, como a presença de doenças neurológicas, músculo-esqueléticas, diabetes, síncopes de origem idiopática, uso de diferentes classes de drogas, incluindo diuréticos, anticonvulsivantes, antihipertensivos, ansiolíticos e antidepressivos $(7,8)$, doenças cardiovasculares e distúrbios metabólicos. Esses dois últimos são os principais responsáveis pelo acometimento do sistema vestibular $(9,10)$.

As doenças cardiovasculares são as principais causas de mortalidade no Brasil sendo que, aproximadamente, $23 \%$ destas, são representadas pelo infarto agudo do miocárdio (IAM) (11). O cateterismo cardíaco de urgência é uma das possibilidades terapêuticas no IAM, com raras complicações (12) como por exemplo, lesão vascular pelo fio guia.

Frente à queixa de vertigem, o médico otorrinolaringologista deve atentar-se para realizar uma boa história clínica e investigar causas, de forma sistemática que justifiquem tal sintomatologia. Desse modo, consegue-se estipular hipóteses diagnósticas plausíveis que podem direcionar, efetivamente, o tratamento. O paciente foi submetido à avaliação clínica otoneurológica, que incluiu anamnese e exame físico otorrinolaringológico, audiometria, imitanciometria e exame vestibular, realizado por intermédio da vectoelectronistagmografia, de acordo com os critérios descritos na literatura (13).

Diante dos achados de anacusia e arreflexia vestibular à direita, o que configura uma cocleo-vestibulopatia severa que poderia envolver alterações da orelha interna, nervos cócleo-vestibulares ou mesmo tronco encefálico, é imprescindível a solicitação de ressonância magnética nuclear para adequado diagnóstico (14). Optou-se pela 


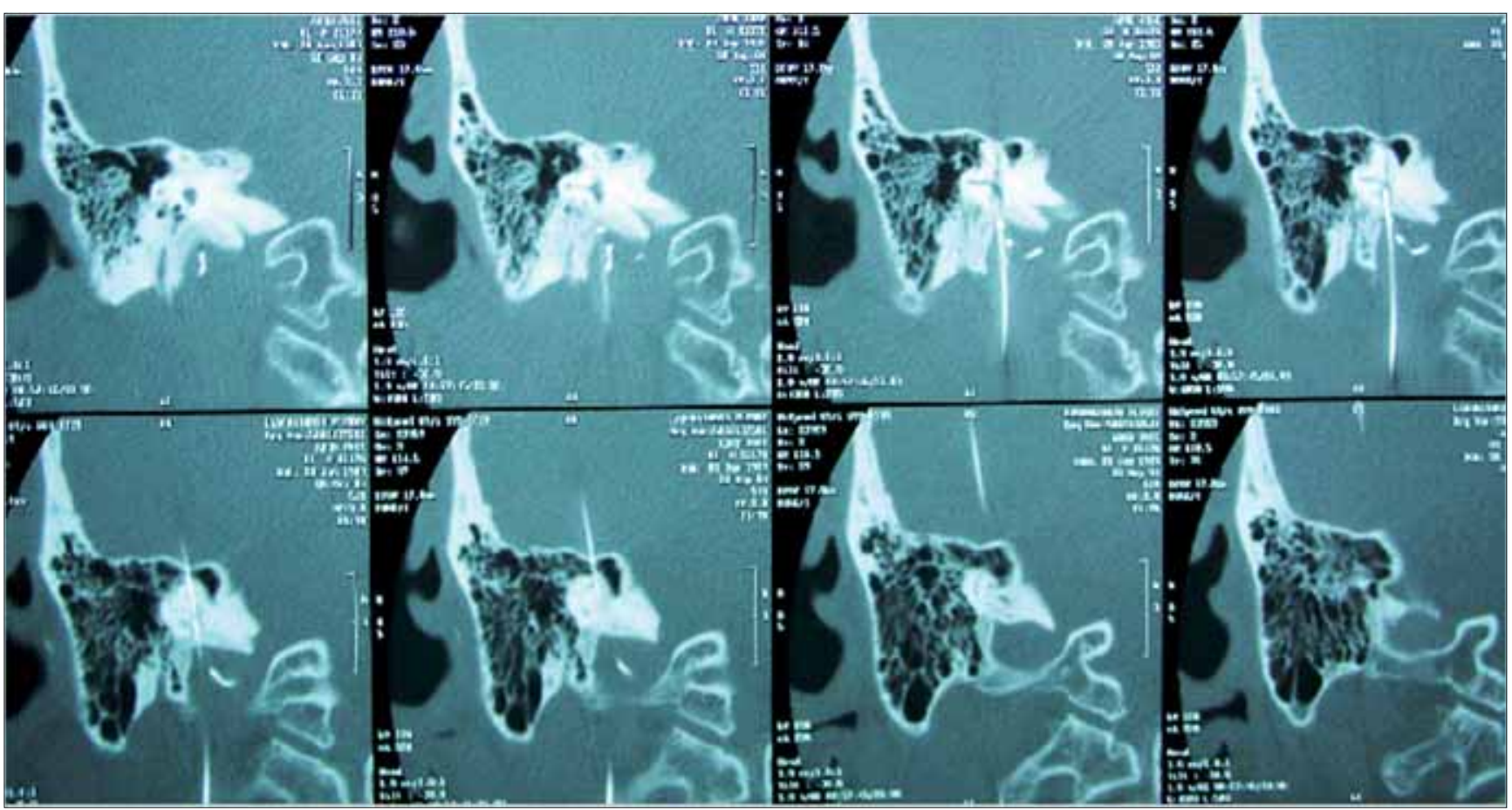

Figura 1. Sequência de imagens de tomografia computadorizada de osso temporal mostrando haste metálica (fio-guia) lesionando os canais semicirculares posterior, superior e vestíbulo, projetando-se em lobo temporal.

tomografia computadorizada (TC) de ossos temporais e tronco-encefálico visto que o paciente apresentou fobia ao entrar no aparelho de ressonância magnética. A TC foi de extrema valia pois fechou o diagnóstico de lesão endovascular após cateterismo de urgência. A avaliação por imagem é utilizada quando a investigação clínica sugere possibilidade de algum comprometimento central responsável pela sintomatologia. A tomografia computadorizada é vantajosa para avaliar alterações ósseas e a ressonância magnética é o melhor método para investigar alterações de partes moles, como por exemplo tumores e degenerações (15).

O paciente foi submetido a avaliações médicas vascular e neurocirúrgica e orientado a controle radiológico do quadro pois a retirada da haste poderia ser extremamente lesiva e ela aparentemente apresentava-se estável no osso não gerando, no momento, riscos de novas lesões endovasculares ou intracranianas. Do ponto de vista otorrinolaringológico, considerando que o paciente era idoso e apresentava uma arreflexia vestibular unilateral de origem traumática, com predisposição à queda, optou-se por terapia de reabilitação vestibular global (RVG) baseada no protocolo de CAWTHORNE e COOKSEY associado a exercícios de adaptação do reflexo vestíbulo-oculomotor. Essas atividades são desenvolvidas através de caminhadas na rua, marcha com abertura e fechamento dos olhos, perseguição de objetos e jogos de bola $(16,17)$. O procedimento foi bem sucedido tendo em vista que o paciente obteve

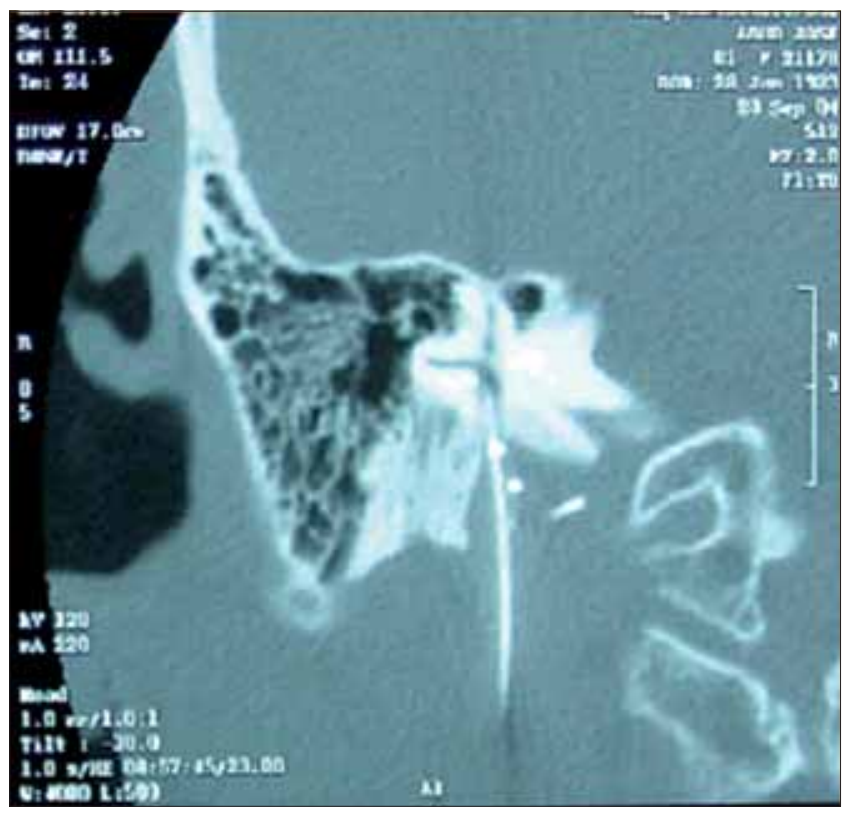

Figura 2. Haste metálica atravessando osso temporal direito à partir da veia jugular interna e bulbo da jugular.

remissão completa dos sintomas ao final de 3 meses de tratamento. Pedalini et al. (18) estudaram 116 pacientes dentre os quais 109 foram analisados quanto ao tempo médio de tratamento para obtenção da melhora. O maior número de pacientes $(n=61)$ necessitou de 2 meses de 
tratamento para referir melhora dos sintomas. Popper (19) afirma que a RVG é um método eficaz, adequado e inócuo; e que os resultados obtidos em seu estudo variaram desde supressão dos sintomas patológicos, restauração do equilíbrio até a provável "cura" objetiva da vestibulopatia.

\section{CONSIDERAÇÕES FINAIS}

Queixas de desequilíbrio no paciente idoso devem ser criteriosamente avaliadas diante do seu histórico clínico patológico pois os antecedentes de doenças e tratamentos prévios, em geral, direcionam as hipóteses diagnósticas porém podem trazer alterações inesperadas.

\section{REFERÊNCIAS BIBLIOGRÁFICAS}

1. Matsumura BA, Ambrose AF. Balance in the Elderly. Clin Geriatr Med. 2006, 22:395-412.

2. Konrad HR, et al. Balance and Aging Triological Society Papers: State Of The Art Review. Laryngoscope. 1999, 109(9):1454-1460.

3. Savitz SI, Caplan LR. Vertebrobasilar Disease. Current Concepts. 2005, 352(25):2618-2626.

4. Simoceli L, et al. Perfil diagnóstico do idoso portador de desequilíbrio corporal: resultados preliminares. Rev Bras Otorrinolaring. 2003, 69:772-777.

5. Bittar RSM, Pedalini MEB, Sznifer J, Formigoni LG. Reabilitação Vestibular: Opção Terapêutica na Síndrome do desequilíbrio do idoso. Gerontologia. 2000, 8(1):9-12.

6. Hirvonen TP, Aalto H, Pyykko I, Juhola M, Jantti P. Changes in vestibulo-ocular reflex of elderly people. Acta Otolaryingol Suppl (Stockh). 1997, 529:108-10.

7. Colledge NR, Wilson JA, MacIntyre CC, MacLennan WJ. The prevalence and characteristics of dizziness in an elderly community. Age Ageing. 1994, 23:117-20.

8. Ensrud KE, Nevitt MC, Yunis C, Hulley SB, Grimm RH, Cummings SR. Postural hypotension and postural dizziness in elderly women. Arch Intern Med. 1992, 152:1058-64.

9. Albernaz PLM. Aspectos otoneurológicos na velhice. Acta Awho. 1982, 1(3):93-5.

10. Ganança MM, et al. As tonturas e sintomas associados In: Munhoz MSL, Caovilla HH, Silva MLG. Casos clínicos otoneurológicos típicos e atípicos. São Paulo: Atheneu; 2001, p. 1-22.

11. Secretaria Vigilância em Saúde - Ministério da Saúde Brasil 2004. www.portal.saude.gov.br/SAUDE/

12. II Diretriz da SBC para tratamento do IAM Arq Bras Cardiol; volume 74, (suplemento II), 2000. www.publicacoes. cardiol.br/consenso/2000/74s2/005.pdf

13. Ganança MM, Caovilla HH, Munhoz MSL, Silva MLG, Frazza MM. As etapas da equilibriometria. In: Ganança MM, Caovilla HH, Munhoz MSL, Silva MLG. Equilibriometria Clínica. Série Otoneurologia. São Paulo: Editora Atheneu; 1999, p. 41-114.

14. Almeida WLC, etal. Vertigem como manifestação isolada de desmielinização no sistema nervoso central: relato de caso e revisão de literatura. Rev Bras Otorrinolaringol. 2004, 70(6):819-822.

15. Menon AD, Sakano E, Weckx LL. Consenso Sobre Vertigem. Rev Bras Otorrinolaringol. 2000, 66(1):9-35.

16. Cooksey FS. Rehabilitation in vestibular injuries. Proc R Soc Med. 1946, 39:273-275.

17. Cawthorne TE. The physiological basis of head exercises. J Chart Soc Physio. Ther. 1944, 29:106-107.

18. Pedalini MEB, Bittar RSM, Formigoni LG, Cruz OLM, Bento RF, Miniti. Reabilitação vestibular como tratamento da tontura: experiência com 116 casos. Arq da Fundação Otorrinolaringologia. 1999, 3(2):74-8.

19. PopperVM. A Reabilitação Vestibular na Vertigem. Centro de Especialização em Fonoaudiologia Clínica, Itajaí, 2001. 